\title{
EL REPARTIMIENTO DEL «SERVICIO Y MEDIO SERVICIO» DE LOS MUDÉJARES DE CASTILLA EN EL ÚLTIMO CUARTO DEL SIGLO XV
}

\author{
GonZalo Viñuales FerReiro
}

UNED, Madrid

Las aljamas y morerías del Reino de Castilla estaban obligadas a pagar desde los tiempos de Juan I, allá por 1388, ciento cincuenta mil maravedíes cada año a la Hacienda Regia en concepto de un impuesto específico, llamado «servicio y medio servicio». Esta carga impositiva, de escasa relevancia en el conjunto de la fiscalidad castellana, gravaba exclusivamente a las minorías étnico-religiosas que habitaban en Castilla. Moros y judíos ingresaban anualmente 600.000 maravedíes en las arcas reales. Como hemos dicho, los mudéjares aportaban 150.000 maravedíes, mientras que la minoría judía contribuía con 450.000 maravedíes ${ }^{1}$. Estas cantidades permanecieron invariables hasta la expulsión de los judíos en 1492, y hasta la conversión en masa de mudéjares de 1502, de tal manera que el valor real del impuesto fue decreciendo a medida que el paso del tiempo trajo aparejada la consiguiente devaluación monetaria. Año tras año, se dividía («repartía») el mencionado montante global entre todas aquellas poblaciones con presencia mudéjar y, en virtud del número de habitantes y de la riqueza de las morerías, se establecía la cantidad que les correspondía tributar.

El repartimiento estaba en manos de una comisión de musulmanes encargada de valorar la realidad mudéjar de Castilla y, a partir de ella, distribuir las cantidades que ese año se debían recaudar. Ya fuera actuando directamente, o bien en nombre de alguno de sus compañeros, las fuentes mencionan a doce recaudadores mudéjares entre 1477 y 1500:

- Maestre Haçán, vecino de Madrid: 1477-1500.

- Maestre Lope, vecino de Madrid: 1477-1486.

- Maestre Hamete Carretón, vecino de Valladolid: 1477-1485.

— Maestre Yuçá de Talavera, vecino de Segovia: 1477-1485.

${ }^{1}$ Para un análisis más detallado de los repartimientos del «servicio y medio servicio» de los judíos de Castilla, remitimos a Ladero Quesada, M. Á.: «Las juderías de Castilla según algunos "servicios" fiscales del siglo XV», Sefarad, XXXI (1971), 249-264 y Viñuales Ferreiro, G.: «Los repartimientos del "servicio y medio servicio" de los judíos de Castilla de 1484, 1485, 1490 y 1491», Sefarad (en prensa).

Al-Qantara XXIV, 1 (2003) 179-202 
- Maestre Mahomad de Talavera, vecino de Segovia, (su hermano): $1477-1485$

- Maestre Farax, vecino de Toledo: 1484-1486.

- Maestre Mahomad Alfaquí, vecino de Segovia: 1486-1493.

- Maestre Abrahem de San Salvador, vecino de Madrid: 1486-1500.

- Maestre Abrahem Maguán, vecino de Toledo: 1493-1494.

- Maestre Abrahem Redomero, vecino de Toledo: 1491-1500.

- Maestre Abrahem Xatafi, vecino de Valladolid: 1493-1500.

— Maestre Yucá de Toledo, vecino de Madrid: 1495-1500.

Estos datos ponen de manifiesto la continuidad en el desempeño de la actividad recaudatoria por parte de un reducido número de arrendadores, entre los que podemos distinguir dos bloques en virtud de dos etapas cronológicas: el primero correspondería al período comprendido entre los años 1477 y 1485, mientras que el segundo desarrollaría sus funciones desde 1486 a 1500. Obsérvese, además, el caso de Maestre Haçán, quien durante veintitrés años ejerció como recaudador del «servicio y medio servicio».

El trabajo realizado por los arrendadores debía ser abonado también por las aljamas y morerías. Así, se establecía que mediante un pago suplementario las aljamas habrían de satisfacer el salario de los recaudadores, que rondaba los 15.000 maravedíes cada año. A su vez se añadía otro pago de menor cuantía, en torno a los 6.000 maravedíes, que tenía por objeto el sufragio de ciertas costas y necesidades de distintas aljamas de Castilla. Esta circunstancia elevaba la cantidad que anualmente desembolsaban las comunidades mudéjares a causa del abono del «servicio y medio servicio» hasta los ciento setenta, o ciento setenta y cinco mil maravedíes anuales.

El «servicio y medio servicio» no fue el único impuesto específico para las minorías judía y mudéjar castellanas. Con motivo de la guerra de Granada, los Reyes Católicos fijaron un nuevo tributo, llamado «los castellanos de oro» por pagarse en esta moneda, equivalente a 485 maravedíes. Tras la toma del reino nazarí, y la expulsión de los judíos, los monarcas mantuvieron esta carga fiscal sobre los mudéjares, que a partir de entonces recibiría el nombre de «pechas». Si comparamos las listas conservadas de «pechas» (1495-1501) con las del «servicio y medio servicio», podemos afirmar que la importancia de este impuesto decreció notablemente, y se mantuvo más por su valor testimonial y tradicional que por su relevancia económica, ya que el montante global de las «pechas» superaba ampliamente al del «servicio y medio servicio». Por ejemplo, en 1495, los mudéjares contribuyeron con 150.000 maravedíes en el pago del «servicio y medio servicio», mientras que en concepto de «pechas» tributaron 1.746 .000 maravedíes, aproximadamente doce veces más.

Sin embargo, la información contenida en estas listas de repartimiento no carece de valor, pues de ellas se pueden extraer ciertas conclusiones acerca del poblamiento y la demografia mudéjar en Castilla a fines del siglo XV. 
Miguel Ángel Ladero Quesada, en sus diversos estudios acerca de la minoría mudéjar que habitaba en territorio castellano ${ }^{2}$, ha planteado ya, a partir de las listas de los repartimientos del «servicio y medio servicio» de los años 1463,1464 y 1501, y de las listas de «pechas» de 1495, 1496, 1498, 1499, 1500 y 1501 , unos sólidos argumentos relacionados con el número de habitantes mudéjares de Castilla, así como con sus áreas de asentamiento y distribución territorial. El estudio de la nueva documentación manejada no viene sino a corroborar los planteamientos entonces expuestos.

En relación a la segunda mitad del siglo XV, no hemos encontrado ninguna evidencia de población mudéjar en el tercio noroccidental de la Península Ibérica, pues las aljamas y morerías más septentrionales son las de Sahagún, Carrión de los Condes, Burgos y Medina de Pomar, aún distantes de las tierras gallegas y cantábricas. Para esas mismas fechas, tampoco ha aparecido mención alguna de comunidades mudéjares en la antigua Extremadura leonesa, lo que ratifica la opinión de que el mudejarismo fue un fenómeno preferentemente castellano y murciano, aunque también se extendió con importancia por el Valle del Guadalquivir y la actual Extremadura.

En el período cronológico que transcurre entre 1477 y 1500, las listas del «servicio y medio servicio» citan 156 poblaciones con habitación mudéjar, aunque es obvio que habría otros lugares con mudéjares entre sus vecinos, como atestigua la repetida frase «e con los que con ellos suelen pechar». Si a esta cifra le añadimos otros 12 lugares que recoge Miguel Ángel Ladero en las listas de las «pechas» (1495-1501) y que no aparecen en el «servicio y medio servicio», resultan 168 poblaciones con presencia mudéjar durante el último cuarto del siglo XV en Castilla.

Sin embargo, este elevado número de morerías y aljamas no debe conducirnos a pensar que la dispersión fue una característica de la minoría mudéjar en la Castilla medieval. Las fuentes nos revelan que la presencia mudéjar en muchos de estos pueblos era modestísima, como en el caso de «el moro de Valdemoro», o de «los dos moros de Coveña, el moro de Algete, el moro de Fuentelsaz, el moro de Paracuellos, y el moro de Talamanca», llegando a ser realmente elocuentes como en las alusiones de «no hay moros salvo dos o tres», o «no hay moros salvo uno pobre». Y también nos descubren los documentos que el poblamiento no fue continuo en algunas comunidades, pues los recaudadores no las contabilizan en algunas listas del «servicio y medio servicio», ya sea porque desaparecieron, o porque pasaron a integrarse en el conjunto del tributo de otra comunidad.

2 Ladero Quesada, M. Á., Los mudéjares de Castilla en tiempo de Isabel I, Valladolid, 1969; «Datos demográficos sobre los musulmanes de Granada y Castilla en el siglo XV», Anuario de Estudios Medievales, 8 (1972-1973); «Los mudéjares de Castilla en la Baja Edad Media», Actas del I Simposio Internacional de Mudejarismo, Madrid-Teruel, 1981, 349-390; y «Los mudéjares en los reinos de la Corona de Castilla. Estado actual de su estudio», Actas III Internacional de Mudejarismo, Teruel, 1986, 5-20. 
Más bien se constata la tendencia opuesta, centrada en una mayor concentración de población en un número bastante reducido de aljamas, pues las cantidades que tributan en concepto de «servicio y medio servicio» superan con un margen muy amplio a las del resto de las comunidades. Y esta realidad se manifiesta en esta misma línea, si nos remitimos a las listas relativas a las «pechas». Por ejemplo, en 1494, las aljamas del territorio comprendido por los obispados de Burgos, Palencia, Ávila y Segovia, abonaron 38.600 maravedíes en concepto de "servicio y medio servicio" de los cuales el $88,6 \%$ fue pagado por las comunidades de mudéjares de Ávila, Segovia, Arévalo y Valladolid, lo que suponía el 19,7\% del total de lo recaudado en Castilla; y al año siguiente, en 1495, de acuerdo al pago de las «pechas», de los 345.320 maravedíes que se pagaron en los dichos obispados, las cuatro aljamas moras ya mencionadas abonaron 168.690 maravedíes, casi el $78 \%$ del total de esos territorios, y el $15,3 \%$ de Castilla.

Respecto a la transcripción de las listas conviene realizar algunas aclaraciones. Con objeto no sólo de facilitar la consulta sino también con el propósito de otorgarle una mayor unidad de contenido, hemos incluido las listas correspondientes a los años 1463, 1464 y 1501 que publicó Ladero Quesa$\mathrm{da}^{3}$. De este citado trabajo también hemos tomado la división regional que el autor estableciera, considerando que responde a la realidad geográfica e histórica de la Castilla de finales del siglo XV. Hemos de advertir también que exclusivamente en el caso del obispado de Cartagena hemos añadido al documento algunas cantidades, escritas en letra cursiva, que no se hallan registradas en él, pero que fueron publicadas por Miguel Rodríguez Llopis ${ }^{4}$. Ahora bien, respecto a este mencionado trabajo debemos indicar que el autor incluye los datos del repartimiento del año 1492 a partir del legajo 51 de la sección de Escribanía Mayor de Rentas. De acuerdo al citado legajo, el único repartimiento que aparece es el correspondiente al año 1493, y así es como nosotros le hemos incluido en el listado. Por último, hay que reseñar que después de cada localidad en el cuadro aparece una letra mayúscula entre paréntesis, indicando la provincia a la que actualmente pertenece cada población de acuerdo al siguiente listado de abreviaturas:
$\mathrm{AB}$
Provincia de Albacete
$\mathrm{AV}$ Provincia de Ávila
BA Provincia de Badajoz
BU Provincia de Burgos
CC Provincia de Cáceres

${ }^{3}$ Ladero Quesada, M. Á., «Los mudéjares de Castilla en la Baja Edad Media», Actas del I Simposio Internacional de Mudejarismo, Madrid, 1981, 383-387.

${ }_{4}$ Rodríguez Llopis, M., «Población y fiscalidad en las comunidades mudéjares del Reino de Murcia (siglo XV)», Actas del III Simposio Internacional de Mudejarismo, Teruel, 1986, 39-53. 
CO Provincia de Córdoba

CR Provincia de Ciudad Real

CU Provincia de Cuenca

GU Provincia de Guadalajara

LE Provincia de León

LR Provincia de La Rioja

M Provincia de Madrid

MU Provincia de Murcia

$P \quad$ Provincia de Palencia

SG Provincia de Segovia

SE Provincia de Sevilla

SO Provincia de Soria

TO Provincia de Toledo

VA Provincia de Valladolid 
[NOTA: Hemos reflejado la cantidad en negrita en aquellas ocasiones en las que la documentación se refiere a la localidad como aljama] [Las llamadas de nota en negrita se refieren a los textos de las tablas y las notas están colocadas al final del documento]

Obispados de Burgos, Palencia, Avila y Segovia

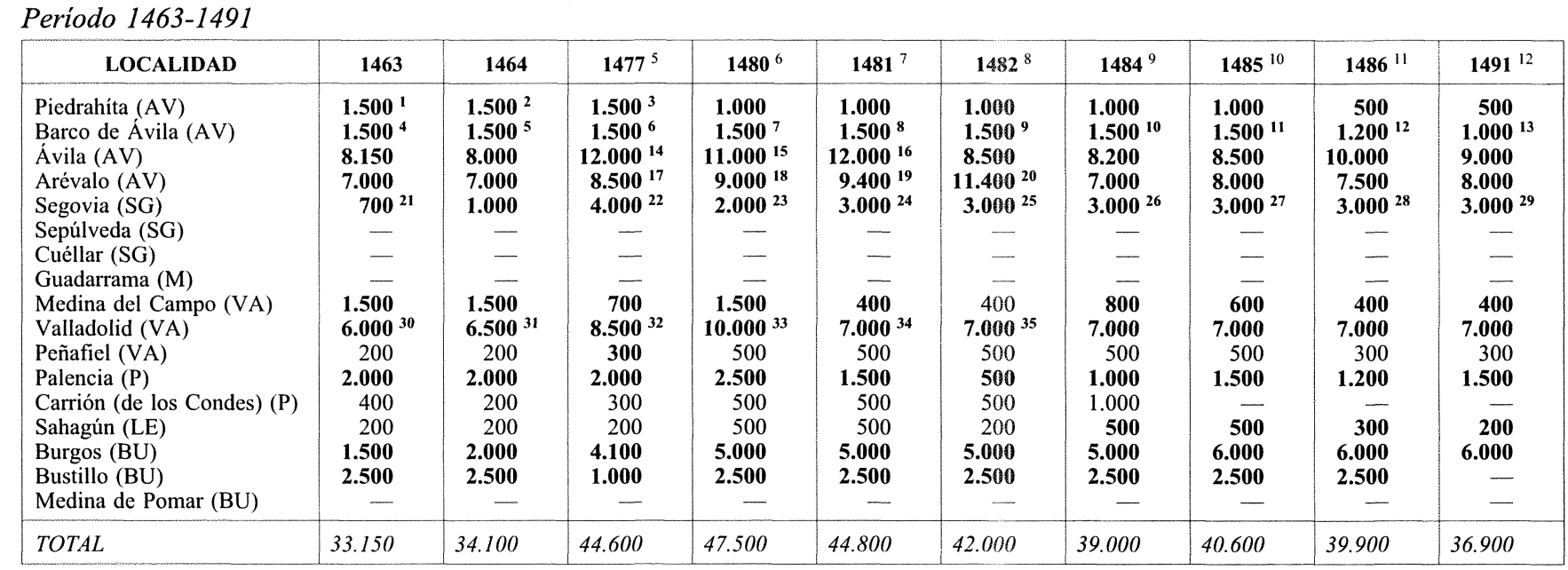

5 Archivo General de Simancas (en adelante, AGS), Escribanía Mayor de Rentas (en adelante, EMR), Legajo 23. Pliegos 167-168.

6 AGS. EMR. Leg. 281. Pliego 305.

AGS. EMR. Leg. 31. Pliego. 291.

AGS. EMR. Leg. 32. Pliego 84.

AGS. EMR. Leg. 36. Pliego 148.

AGS. EMR. Leg. 39. Pliego 490

11 AGS. EMR. Leg. 42. Pliego 273.

12 AGS. EMR. Leg. 50. Pliego 270. 


\begin{tabular}{|c|c|c|c|c|c|c|c|c|c|}
\hline LOCALIDAD & $1493^{13}$ & $1494^{14}$ & $1495^{15}$ & $1496^{16}$ & $1497^{17}$ & $1498^{18}$ & $1499^{19}$ & $1500^{20}$ & 1501 \\
\hline Piedrahíta (AV) & 500 & 500 & 600 & 600 & 600 & 600 & 600 & 600 & 600 \\
\hline Barco de Avila (AV) & 500 & $500^{36}$ & 600 & 600 & 600 & 600 & 600 & 600 & 600 \\
\hline Ávila (AV) & $13.000^{37}$ & $13.000^{38}$ & $12.000^{39}$ & $12.500^{40}$ & $11.200^{41}$ & $11.500^{42}$ & $11.500^{43}$ & $11.500^{44}$ & 11.500 \\
\hline Arévalo (AV) & 8.700 & 8.700 & 8.700 & 8.700 & 8.000 & 8.500 & 8.900 & 10.000 & 10.000 \\
\hline Segovia (SG) & $\mathbf{5 . 5 0 0}$ & 5.500 & $3.500^{45}$ & $4.000^{46}$ & $3.500^{47}$ & $3.500^{48}$ & $3.500^{49}$ & $4.000^{50}$ & $4.000^{51}$ \\
\hline Sepúlveda (SG) & - & - & - & - & - & 300 & 300 & 300 & 300 \\
\hline Cuéllar (SG) & 300 & $200^{52}$ & - & - & - & & - & - & - \\
\hline Guadarrama (M) & 500 & $500^{53}$ & - & - & - & - & - & - & - \\
\hline Medina del Campo (VA) & 200 & 200 & 300 & 200 & 300 & 300 & 300 & 300 & 300 \\
\hline Valladolid (VA) & 7.000 & 7.000 & 7.000 & 7.000 & 7.000 & 7.000 & 7.000 & 7.000 & 7.000 \\
\hline Peñafiel (VA) & 300 & & & & & & & & \\
\hline Palencia (P) & 2.000 & 1.000 & 1.000 & 1.000 & 1.500 & 1.100 & 1.400 & 1.600 & 1.600 \\
\hline Carrión (de los Condes) (P) & - & & - & - & - & 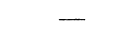 & - & - & - \\
\hline Sahagún (LE) & - & - & - & - & - & - & - & - & - \\
\hline Burgos (BU) & - & - & - & - & - & - & - & - & - \\
\hline Bustillo (BU) & 1.500 & 1.500 & $\overline{-}$ & $\overline{-}^{55}$ & $\sqrt{500^{56}}$ & $-\overline{-}$ & $\sqrt{500} 58$ & $2500^{59}$ & $2500^{60}$ \\
\hline Medina de Pomar (BU) & - & - & $1.500^{54}$ & $1.500^{55}$ & $1.500^{56}$ & $1.500^{57}$ & $2.500^{58}$ & $2.500^{59}$ & $2.500^{60}$ \\
\hline TOTAL & 40.000 & 38.600 & 35.200 & 36.100 & 34.200 & 34.900 & 36.600 & 38.400 & 38.400 \\
\hline
\end{tabular}

13 AGS. EMR. Leg. 51. Pliego 273.

${ }^{4}$ AGS. EMR. Leg. 55. Pliegos 494-495.

5 AGS. EMR. Leg. 58. Pliegos 5-6.

${ }^{16}$ AGS. EMR. Leg. 60. Pliegos 811-812.

AGS. EMR. Leg. 62 ${ }^{2}$. Pliegos $984-985$ y $988-990$.

${ }_{18}$ AGS. EMR. Leg. 62². Pliegos 986-987.

19 AGS. EMR. Leg. 69. Pliegos 563-566. 
Periodo: 1493-1501

\begin{tabular}{|c|c|c|c|c|c|c|c|c|c|}
\hline LOCALIDAD & 1493 & 1494 & 1495 & 1496 & 1497 & 1498 & 1499 & 1500 & 1501 \\
\hline $\begin{array}{l}\text { Sigüenza (GU) } \\
\text { Xuera }\end{array}$ & - & - & - & E & - & - & - & - & - \\
\hline Ágreda (SO) & 6.000 & 5.000 & 5.000 & 6.000 & 6.000 & 6.000 & 6.000 & 6.000 & 6.000 \\
\hline Ayllón (SG) & 800 & 800 & 1.000 & 1.000 & 1.000 & 1.000 & 1.000 & 1.000 & 1.000 \\
\hline Cervera (LR) & 800 & 800 & 1.000 & 1.000 & 1.000 & 1.000 & 1.000 & 1.000 & 1.000 \\
\hline Aranda de Duero (BU) & 1.500 & 1.500 & 1.500 & 2.000 & 2.000 & 1.800 & 1.900 & 2.000 & 2.000 \\
\hline Peñaranda de Duero (BU) & 600 & 600 & 600 & 600 & 500 & 600 & 600 & 600 & 600 \\
\hline Berlanga de Duero (SO) & & & 500 & 500 & 500 & 500 & 500 & 500 & 500 \\
\hline S. Esteban de Gormaz (SO) & 300 & 300 & 400 & 400 & 400 & 400 & 400 & 400 & 400 \\
\hline Aguilar de Cervera (LR) & 2.000 & 2.000 & 2.500 & 2.500 & 2.500 & 2.500 & 2.500 & 2.500 & 2.500 \\
\hline Medinaceli (SO) & 2.500 & 2.500 & 2.500 & 2.500 & 2.500 & & & & \\
\hline Molina de Aragón (GU) & 1.000 & 1.000 & 1.500 & 1.500 & 1.500 & 1.500 & 1.300 & 1.300 & 1.300 \\
\hline Deza (SO) & 1.800 & 1.800 & 2.000 & 2.000 & 2.000 & 2.000 & 2.000 & 2.000 & 2.000 \\
\hline Castillejos & - & - & & & & - & & & - \\
\hline Préjano (LR) & - & - & $1.500^{98}$ & 1.500 & 1.10099 & & $1.500^{100}$ & $1.500^{101}$ & \\
\hline Herce (LR) & $1.650^{102}$ & $1.600^{103}$ & 500 & 500 & & 1.500 & & & $1.500^{104}$ \\
\hline $\operatorname{Arcos}(\mathrm{SO})$ & 1.800 & 1.800 & 1.500 & & 1.500 & 1.500 & 1.500 & 1.500 & 1.500 \\
\hline Haro (LR) & & & 500 & 500 & $\mathbf{5 0 0}$ & 500 & & & \\
\hline Nájera (LR) & 500 & 500 & 300 & 300 & 300 & 500 & 500 & 500 & 500 \\
\hline Arnedo (LR) & 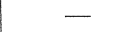 & - & - & - & 600 & - & 500 & 500 & 500 \\
\hline Belorado (BU) & - & - & - & - & $300^{105}$ & - & - & - & - \\
\hline TOTAL & 21.250 & 20.200 & 22.800 & 22.800 & 24.200 & 21.300 & 21.200 & 21.300 & 21.300 \\
\hline
\end{tabular}


Arzobispado de Toledo y Obispado de Cuenca

\begin{tabular}{|c|c|c|c|c|c|c|c|c|c|c|}
\hline LOCALIDAD & 1463 & 1464 & 1477 & 1480 & 1481 & 1482 & 1484 & 1485 & 1486 & 1491 \\
\hline Toledo (TO) & $\begin{array}{r}8.500^{106} \\
7116\end{array}$ & $\begin{array}{r}8.500^{107} \\
400117\end{array}$ & $\begin{array}{r}9.500^{108} \\
350^{118}\end{array}$ & $\begin{array}{r}8.500109 \\
600119\end{array}$ & $\begin{array}{r}8.500110 \\
300120\end{array}$ & $\begin{array}{r}10.000^{111} \\
30{ }^{121}\end{array}$ & $\begin{array}{r}8.000^{112} \\
300^{122}\end{array}$ & $8.000^{113}$ & $8.000^{114}$ & $8.000^{115}$ \\
\hline Madrid (M) & $1.000^{125}$ & $1.000^{126}$ & $\begin{array}{r}3500^{127} \\
1.507\end{array}$ & $1.500^{128}$ & $\begin{array}{r}300 \\
1.500^{129}\end{array}$ & $1.500^{\mathbf{1 3 0}}$ & $1.500^{131}$ & $1.500^{132}$ & $1.100^{133}$ & $1.000^{134}$ \\
\hline Chinchón $(\mathrm{M})^{25}$ & - & & & & & & & & & \\
\hline Alcalá de Henares (M) & $800^{135}$ & $1.200^{1}$ & 1.500 & $1.500^{138}$ & 600 & $1.100^{140}$ & $500^{1}$ & $500^{142}$ & $500^{143}$ & 500 \\
\hline Guadalajara (GU) & $7.500^{144}$ & $7.000^{145}$ & $7.500^{146}$ & $7.000^{147}$ & $7.500^{148}$ & $7.500^{149}$ & 7.500 & $7.500^{150}$ & $7.000^{151}$ & 7.500 \\
\hline Talavera de la Reina (TO) & $1.000^{152}$ & $1.000^{153}$ & $2.000^{154}$ & $2.000^{155}$ & $2.000^{156}$ & $2.500^{157}$ & 2.000158 & $2.000^{159}$ & 2.200 & 2.000 \\
\hline Hita (GU) & 1.500160 & $1.500^{161}$ & $1.500^{162}$ & $1.000^{163}$ & $1.000^{164}$ & $1.000^{165}$ & $1.000^{166}$ & $600^{167}$ & $600^{168}$ & 300 \\
\hline Huete $(\mathrm{CU})$ & 1.600169 & $1.200^{170}$ & $1.500^{171}$ & $1.500^{172}$ & $1.500^{173}$ & $1.500^{174}$ & $1.200^{175}$ & $1.200^{176}$ & $1.300^{177}$ & $1.500^{178}$ \\
\hline Cuenca (CU) & $2.000^{179}$ & $1.500^{180}$ & $1.500^{181}$ & $800^{182}$ & $1.000^{183}$ & $1.300^{184}$ & 600 & $600^{185}$ & $700^{186}$ & $600^{187}$ \\
\hline El Congosto $(\mathrm{CU})$ & $800^{188}$ & $1.000^{189}$ & 1.500190 & $300^{191}$ & $300^{192}$ & $300^{193}$ & $600^{194}$ & $300^{195}$ & $500^{196}$ & $500^{197}$ \\
\hline Escalona (TO) & 2.000 & 2.000 & 2.000 & 1.500 & 1.500 & 1.500 & 1.000 & 1.000 & 1.000 & 600 \\
\hline San Clemente (CU) & $600^{198}$ & $800^{199}$ & $500^{200}$ & $300^{201}$ & $300^{202}$ & $300^{203}$ & $500^{204}$ & $500^{205}$ & $600^{206}$ & $800^{207}$ \\
\hline Belmo & 600 & 600 & & 500 & 500 & 500 & 300 & 300 & 500 & 300 \\
\hline Castillo de Garci Muñ & - & 1.000 & 1.500 & 500 & 500 & 500 & 500 & 500 & 700 & 800 \\
\hline TOTAL & 28.600 & 28.700 & 32.350 & 27.500 & 27.000 & 29.800 & 25.500 & 24.800 & 25.100 & 24.600 \\
\hline
\end{tabular}

裀

${ }^{25}$ Véase las notas del final números 22 y 26. 


\begin{tabular}{|c|c|c|c|}
\hline \multicolumn{4}{|c|}{$A Q, \mathrm{XXIV}, 2003$} \\
\hline & $\underline{\overline{\mathbf{n}}}$ & 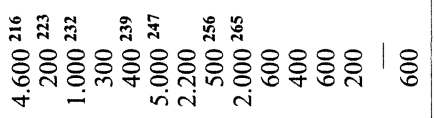 & $\begin{array}{c}8 \\
0 \\
0 \\
0\end{array}$ \\
\hline & เิ & 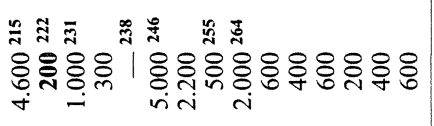 & $\mid \begin{array}{c}8 \\
0 \\
0 \\
0\end{array}$ \\
\hline & $\frac{2}{\partial}$ & 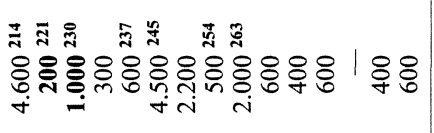 & $\mid \begin{array}{l}8 \\
0 \\
0 \\
0\end{array}$ \\
\hline & $\begin{array}{l}\infty \\
\stackrel{\alpha}{q}\end{array}$ & 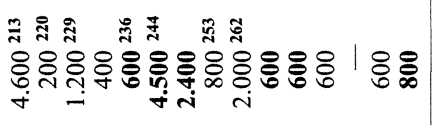 & $\begin{array}{c}8 \\
\vdots \\
2\end{array}$ \\
\hline & $\underline{G}$ & 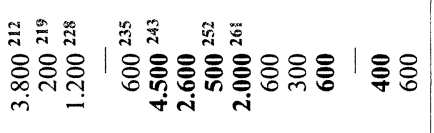 & \begin{tabular}{c}
8 \\
$\vdots$ \\
\hdashline
\end{tabular} \\
\hline & $\underset{\sigma}{\stackrel{\sigma}{\sigma}}$ & 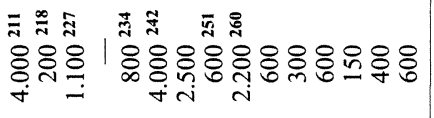 & $\begin{array}{c}0 \\
8 \\
0 \\
0\end{array}$ \\
\hline & $\stackrel{\stackrel{\circ}{g}}{\mathrm{~g}}$ & 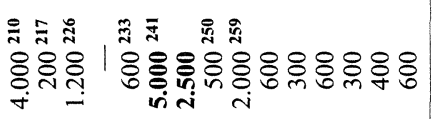 & $\begin{array}{l}8 \\
\infty \\
\infty \\
0\end{array}$ \\
\hline & 夺 & 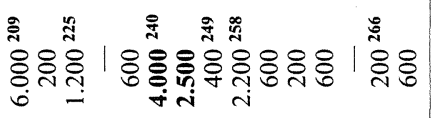 & $\begin{array}{c}8 \\
2 \\
2\end{array}$ \\
\hline & $\frac{\tilde{g}}{q}$ & 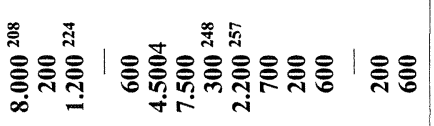 & $\begin{array}{l}8 \\
0 \\
0 \\
0\end{array}$ \\
\hline 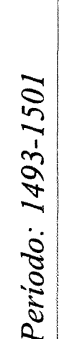 & 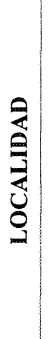 & 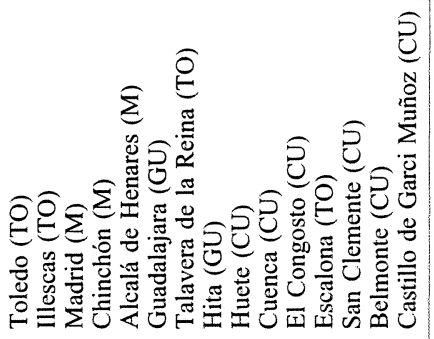 & 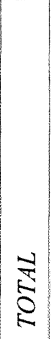 \\
\hline
\end{tabular}


Obispados de Coria, Plasencia y Badajoz

\begin{tabular}{|c|c|c|c|c|c|c|c|c|c|c|}
\hline LOCALIDAD & 1463 & 1464 & 1477 & 1480 & 1481 & 1482 & 1484 & 1485 & 1486 & 1491 \\
\hline $\begin{array}{l}\text { Trujillo (CC) } \\
\text { Plasencia (CC) } \\
\text { Medellín (BA) }\end{array}$ & $\begin{array}{l}3.500 \\
1.500 \\
2.800\end{array}$ & $\begin{array}{l}2.500 \\
1.500 \\
2.800\end{array}$ & $\begin{array}{l}\mathbf{3 . 0 0 0}^{267} \\
\mathbf{2 . 0 0 0}^{271} \\
\mathbf{2 . 0 0 0}\end{array}$ & $\begin{array}{l}\mathbf{5 . 0 0 0} \\
\mathbf{4 . 5 0 0}^{272} \\
\mathbf{2 . 5 0 0}^{278}\end{array}$ & $\begin{array}{l}5.500 \\
4^{4.000} \\
2.000^{273}\end{array}$ & $\begin{array}{l}5.500 \\
5.000^{274} \\
2.000^{280}\end{array}$ & $\begin{array}{l}4.500 \\
4.000 \\
1.000\end{array}$ & $\begin{array}{l}6.000 \\
4.500 \\
300\end{array}$ & $\begin{array}{r}4.500 \\
4.500 \\
-\end{array}$ & $\begin{array}{l}6.000{ }^{268} \\
4.500\end{array}$ \\
\hline TOTAL & 7.800 & 6.800 & 7.000 & 12.000 & 11.500 & 12.500 & 9.500 & 10.800 & 9.000 & 10.500 \\
\hline
\end{tabular}

Periodo: $1493-1500$

\begin{tabular}{|l|c|c|c|c|c|c|c|c|c|c|}
\hline \multicolumn{1}{|c|}{ LOCALIDAD } & $\mathbf{1 4 9 3}$ & $\mathbf{1 4 9 4}$ & $\mathbf{1 4 9 5}$ & $\mathbf{1 4 9 6}$ & $\mathbf{1 4 9 7}$ & $\mathbf{1 4 9 8}$ & $\mathbf{1 4 9 9}$ & $\mathbf{1 5 0 0}$ & $\mathbf{1 5 0 1}$ \\
\hline Trujillo (CC) & $\mathbf{7 . 1 0 0} 281$ & $\mathbf{7 . 0 0 0} 282$ & $\mathbf{7 . 0 0 0} 283$ & $\mathbf{7 . 4 5 0} 284$ & $\mathbf{7 . 0 0 0} 285$ & $\mathbf{6 . 5 0 0} 286$ & $\mathbf{7 . 0 0 0} 287$ & $\mathbf{6 . 5 0 0}$ & $\mathbf{6}$ \\
Plasencia (CC) & $\mathbf{6 . 0 0 0} 289$ & $\mathbf{6 . 0 0 0} 290$ & $\mathbf{6 . 0 0 0}$ & $\mathbf{6 . 0 0 0}$ & $\mathbf{5 . 5 0 0}$ & $\mathbf{5 . 0 0 0}$ & $\mathbf{4 . 7 0 0}$ & $\mathbf{4 . 7 0 0}$ & $\mathbf{4 . 7 0 0}$ \\
Medellín (BA) & - & - & 200 & 200 & 200 & $\mathbf{2 0 0}$ & 200 & 200 & 200 \\
\hline TOTAL & 13.100 & 13.000 & 13.200 & 13.650 & 12.700 & 11.700 & 11.900 & 11.400 & 11.400 \\
\hline
\end{tabular}


Arzobispado de Sevilla, y Obispados de Cádiz, Córdoba y Jaén Periodo: 1463-1491

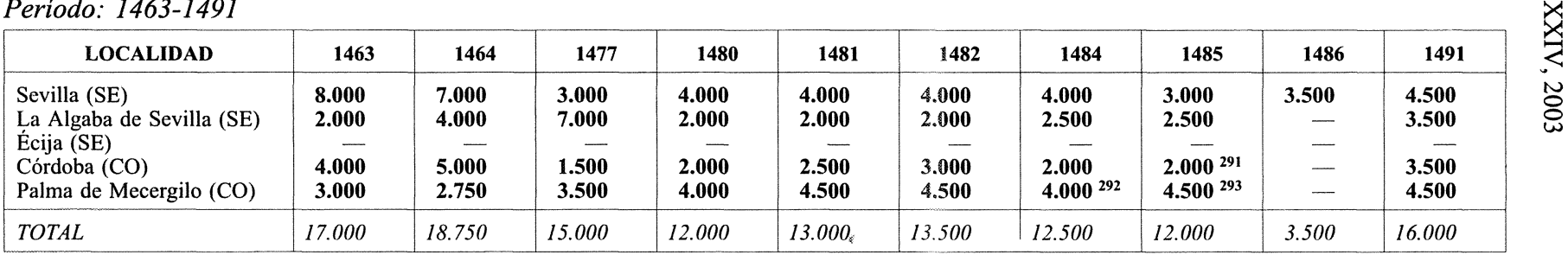

Periodo: $1493-1500$

\begin{tabular}{|l|c|c|c|c|c|c|c|c|c|}
\hline \multicolumn{1}{|c|}{ LOCALIDAD } & $\mathbf{1 4 9 3}$ & $\mathbf{1 4 9 4}$ & $\mathbf{1 4 9 5}$ & $\mathbf{1 4 9 6}$ & $\mathbf{1 4 9 7}$ & $\mathbf{1 4 9 8}$ & $\mathbf{1 4 9 9}$ & $\mathbf{1 5 0 0}$ & $\mathbf{1 5 0 1}$ \\
\hline Sevilla (SE) & $\mathbf{4 . 5 0 0}$ & $\mathbf{4 . 5 0 0}$ & $\mathbf{4 . 0 0 0}$ & 4.000 & $\mathbf{4 . 0 0 0}$ & $\mathbf{4 . 0 0 0}$ & 4.000 & 4.000 & 4.000 \\
La Algaba de Sevilla (SE) & $\mathbf{2 . 2 5 0}$ & $\mathbf{2 . 5 0 0}$ & $\mathbf{2 . 5 0 0}$ & 2.500 & $\mathbf{2 . 5 0 0}$ & 3.200 & 2.500 & 2.500 & 2.500 \\
Écija (SE) & - & $\mathbf{5 0 0}$ & $\mathbf{5 0 0}$ & 500 & -500 & 500 & 600 & 600 & 600 \\
Córdoba (CO) & $\mathbf{3 . 0 0 0}$ & $\mathbf{3 . 0 0 0}$ & $\mathbf{3 . 0 0 0}$ & 3.000 & $\mathbf{3 . 0 0 0}$ & 3.000 & 4.000 & 3.000 & 3.000 \\
Palma de Mecergilo ${ }^{26}$ (CO) & $\mathbf{3 . 3 0 0}$ & $\mathbf{4 . 3 0 0}$ & $\mathbf{4 . 5 0 0}$ & 4.500 & $\mathbf{4 . 5 0 0}$ & $\mathbf{4 . 5 0 0}$ & 4.500 & 4.500 & 4.500 \\
\hline TOTAL & 13.050 & 14.300 & 14.500 & 14.500 & 14.000 & 15.200 & 15.600 & 14.600 & 14.600 \\
\hline
\end{tabular}

${ }^{26} \mathrm{La}$ antigua localidad de Palma de Mecergilo es en la actualidad la villa cordobesa de Palma del Río. Recibía ese nombre por pertenecer al lugar de Micer Egidio Bocanegra. 
Obispado de Cartagena

Periodo: 1463-1491

\begin{tabular}{|c|c|c|c|c|c|c|c|c|c|c|}
\hline LOCALIDAD & 1463 & 1464 & 1477 & 1480 & 1481 & 1482 & 1484 & 1485 & 1486 & 1491 \\
\hline Murcia (MU) & 6.000 & 5.500 & $3.500^{294}$ & $5.000^{295}$ & $5.000^{296}$ & $5.000^{297}$ & 4.000 & 4.000 & - & 5.500 \\
\hline Alcantarilla (MU) & 3.500 & 3.500 & 5.000 & 6.500 & 7.000 & 7.000 & 7.000 & 7.000 & - & $\mathbf{7 . 5 0 0}$ \\
\hline La Puebla del Doctor de Cascales ${ }^{27}$ (MU) & 2.000 & 2.000 & 2.000 & 2.000 & 2.500 & 2.500 & 3.000 & 3.000 & - & 3.500 \\
\hline Abanilla (MU) & 2.000 & 2.000 & 4.000 & 6.000 & 6.500 & 6.500 & 6.500 & 6.500 & - & 2.000 \\
\hline Alguaza del Obispo ${ }^{28}(\mathrm{MU})$ & 2.000 & 2.000 & 2.000 & 2.500 & 3.000 & 3.000 & 3.000 & 3.000 & - & 3.500 \\
\hline Albudeite (MU) & & & & & & & & & - & 1.500 \\
\hline Archena (MU) & 500 & 500 & 2.000 & 1.500 & 1.500 & 1.500 & 2.500 & 2.500 & - & 2.500 \\
\hline San Martín (MU) & - & - & - & - & - & - & - & - & - & - \\
\hline Fortuna (MU) & - & - & - & - & - & - & - & - & - & 3.000 \\
\hline Campos (MU) & - & - & & & - & - & & - & - & 2.000 \\
\hline Molina (MU) & 5.000 & 5.000 & 6.000 & 5.000 & 5.000 & 5.000 & 7.000 & 5.000 & - & 5.000 \\
\hline $\begin{array}{l}\text { Avellán }{ }^{29} \\
\text { (2) }\end{array}$ & & & & & & & & & & \\
\hline Añora ${ }^{30}$ (MU) & 200 & 200 & 1.000 & 1.000 & 1.000 & 1.000 & 900 & 900 & - & 1.500 \\
\hline Puebla Nueva (MU & - & - & & 2.000 & 2.000 & 2.000 & 2.000 & 2.000 & - & 500 \\
\hline Puebla de Fajardo ${ }^{31}$ (MU) & - & - & 2.000 & - & - & - & - & - & - & - \\
\hline Monteagudo (MU) & - & - & 1.000 & - & - & - & - & - & - & - \\
\hline Cotillas (MU) & - & - & & - & - & - & - & - & - & 2.000 \\
\hline Puebla de Mula (MU) & - & - & 2.000 & 2.000 & 500 & - & - & - & - & - \\
\hline Vuéllar ${ }^{32}$ & - & - & 500 & 500 & - & - & - & - & - & - \\
\hline Vélez ${ }^{33}$ & - & - & & - & - & - & - & - & - & - \\
\hline Cieza (MU) & - & - & 3.000 & - & - & - & - & - & - & - \\
\hline Hellin (AB) & 2.000298 & 2.000 & 3.000 & 1.000 & - & - & - & - & - & - \\
\hline TOTAL & 23.200 & 22.700 & 37.000 & 35.000 & 34.000 & 33.500 & 35.900 & 33.900 & - & 40.000 \\
\hline
\end{tabular}

27 Esta localidad es la misma que la de Puebla de Soto (prov. de Murcia).

${ }^{28}$ El nombre contemporáneo de esta población es el de Alguazas (prov. de Murcia)

${ }^{29}$ No hemos encontrado la localidad actual de dicha población, aunque al pechar con el Palomar, la ubicamos en la provincia de Murcia.

30 Actualmente esta localidad se denomina La Nora (prov. de Murcia).
31 La Puebla de Fajardo se corresponde con la localidad de Torre de Juan Vicente, y con Palomar de Juan Vicente, según Juan Torres Fontes en «Los mudéjares murcianos: economía y sociedad», en Actas del IV Simposio Internacional de Mudejarismo, Teruel, 1992, pp. 365-393. 32 Idem.

33 Esta localidad la recoge Miguel Ángel Ladero Quesada como Vélez. Pensamos que ha de hacer referencia a una de las actuales localidades granadinas, muy próximas al límite provincial murciano, de Vélez Rubio, o Vélez Blanco. 
Período: 1493-1501

\begin{tabular}{|c|c|c|c|c|c|c|c|c|c|}
\hline LOCALIDAD & 1493 & 1494 & 1495 & 1496 & 1497 & 1498 & 1499 & 1500 & 1501 \\
\hline $\begin{array}{l}\text { Murcia (MU) } \\
\text { Alcantarilla (MU) }\end{array}$ & 6.000 & 6.000 & 5.000 & 5.000 & 6.000 & 6.000 & 6.000 & 4.000 & 4.000 \\
\hline $\begin{array}{l}\text { Alcantarilla (MU) } \\
\text { La Puebla del Doctor de Cascales (MU) }\end{array}$ & $\begin{array}{l}8.500 \\
4.000\end{array}$ & $\begin{array}{l}8.500 \\
4.000\end{array}$ & 6.000 & 7.500 & 7.500 & 7.000 & 7.000 & 6.500 & 6.500 \\
\hline Abanilla (MU) & & & - & - & - & - & - & - & 4.000 \\
\hline Alguaza del Obispo (MU) & 4.000 & 4.000 & 2.000299 & 2.500300 & $2.500^{301}$ & 2.500 & $2.500^{302}$ & 2.200 & 2.200 \\
\hline Albudeite (MU) & 1.700 & 1.800 & 2.000 & 1.700 & 1.700 & 1.700 & 1.900 & 1.700 & 1.700 \\
\hline Archena (MU) & 1.800 & 1.800 & 2.000 & 1.600 & 1.600 & 1.600 & 1.600 & 1.600 & 1.600 \\
\hline San Martín (MU) & & & 200 & 100 & 100 & 100 & 100 & 100 & 100 \\
\hline Fortuna (MU) & 3.300 & 3.300 & 1.500 & 1.800 & 2.000 & 1.800 & 1.900 & 1.800 & 1.800 \\
\hline Campos (MU) & 2.350 & 2.300 & 2.000 & 1.700 & 1.700 & 1.700 & 1.900 & 1.700 & 1.700 \\
\hline Molina (MU) & 4.000 & 4.000 & 5.000 & 5.000 & 5.000 & 5.000 & 5.000 & 5.000 & 5.000 \\
\hline Avellán & & & $300^{303}$ & $200^{304}$ & $200^{305}$ & 200 & 200 & & \\
\hline Añora (MU) & $200^{306}$ & 300 & 300 & 300 & 300 & 300 & 300 & 300 & 300 \\
\hline Puebla Nueva (MU) & 1.200 & 200 & 4.000 & 3.700 & 3.700 & 3.700 & 3.700 & 4.000 & - \\
\hline Puebla de Fajardo (MU) & - & - & - & - & & - & - & - & - \\
\hline Monteagudo (MU) & - & - & - & - & - & - & - & - & - \\
\hline Cotillas (MU) & $500^{307}$ & 600 & - & - & - & - & - & - & - \\
\hline Puebla de Mula (MU) & - & - & 400 & 200 & 200 & 400 & 400 & 400 & 400 \\
\hline Vuéllar & - & - & & & - & & & $\pi$ & \\
\hline Vélez & - & - & - & - & - & - & - & - & 3.300 \\
\hline Cieza (MU) & - & - & - & - & - & - & - & 200 & 200 \\
\hline Hellin (AB) & - & - & - & - & 1.000 & 1.000 & 1.000 & 1.000 & 1.000 \\
\hline TOTAL & 37.550 & 36.800 & 30.700 & 31.300 & 33.500 & 33.000 & 33.500 & 30.500 & 33.800 \\
\hline
\end{tabular}

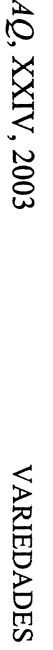


Órdenes Militares

Periodo: $1463-1491$

\begin{tabular}{|c|c|c|c|c|c|c|c|c|c|c|}
\hline LOCALIDAD & 1463 & 1464 & 1477 & 1480 & 1481 & 1482 & 1484 & 1485 & 1486 & 1491 \\
\hline Ceuti del Bachiller ${ }^{34}$ (MU) & 2.500 & 2.500 & $\mathbf{2 . 0 0 0}$ & 2.000 & 2.000 & 2.000 & 2.000 & 2.000 & - & 2.000 \\
\hline Val de Ricote (MU) & - & - & 15.000308 & 2.000 & 2.000 & 2.000 & 1.000 & 1.000 & - & 1.000 \\
\hline Lorquí (MU) & - & - & 3.000 & 500 & 500 & 500 & $\mathbf{5 0 0}$ & 500 & 500 & 300 \\
\hline Socovos (AB) & - & - & 1.000 & 200 & 200 & 200 & 200 & 200 & - & 200 \\
\hline Pliego (MU) & - & - & 2.000 & 300 & 300 & 300 & 300 & 300 & - & 200 \\
\hline Ocaña (TO) & - & - & 2.000 & 500 & 500 & 500 & 500 & 500 & - & 500 \\
\hline Uclés (CU) & - & - & 10.000 & 1.500 & 1.500 & 1.500 & 1.000 & 1.000 & - & 1.000 \\
\hline Hornachos (BA) & - & - & 20.000 & 6.000 & 6.000 & 6.000 & 7.000 & 7.000 & - & 7.000 \\
\hline Llerena (BA) & - & - & 2.000 & $\mathbf{5 0 0}$ & 500 & 500 & $\mathbf{5 0 0}$ & $\mathbf{5 0 0}$ & - & $\mathbf{5 0 0}$ \\
\hline Magacela (BA) & - & - & & 500 & 500 & 500 & $\mathbf{5 0 0}$ & 500 & - & 500 \\
\hline Mérida (BA) & - & - & 2.000 & 500 & 500 & 500 & & & - & \\
\hline Bienquerencia (BA) & - & - & & 500 & 500 & 500 & 500 & 500 & - & 500 \\
\hline Burguillos ${ }^{35}$ (BA) & - & - & $3.000^{309}$ & - & - & - & - & - & - & - \\
\hline Alcántara (CC) & - & - & $-\overline{2}$ & - & 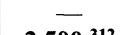 & - & 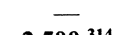 & - & - & - \\
\hline Villarrubia de Arenas (TO) & - & - & $3.000^{310}$ & $2.000^{311}$ & $2.500^{312}$ & $2.500^{313}$ & $2.500^{314}$ & $2.500^{315}$ & $1.500^{316}$ & $2.200^{317}$ \\
\hline Aldea del Rey (CR) & - & - & 3.000 & 2.000 & 2.000 & 2.000 & 2.000 & 1.600 & 1.500 & 1.500 \\
\hline Almagro (CR) & - & - & - & - & - & - & - & - & - & - \\
\hline Almadén (CR) & - & - & - & - & - & - & - & - & - & - \\
\hline Daimiel (CR) & - & - & - & - & - & - & - & - & - & - \\
\hline Montiel (CR) & - & - & 3.000 & - & - & - & - & - & - & - \\
\hline TOTAL & $(2.500)$ & $(2.500)$ & 71.000 & 19.000 & 19.500 & 19.500 & 18.500 & 18.100 & $(3.500)$ & 17.400 \\
\hline
\end{tabular}

34 Esta localidad se llama en la actualidad Cebtí (prov. de Murcia).

35 Consideramos que en la actualidad se corresponde con la localidad de Burguillos del Cerro (prov. de Badajoz). 
Periodo: 1493-1501

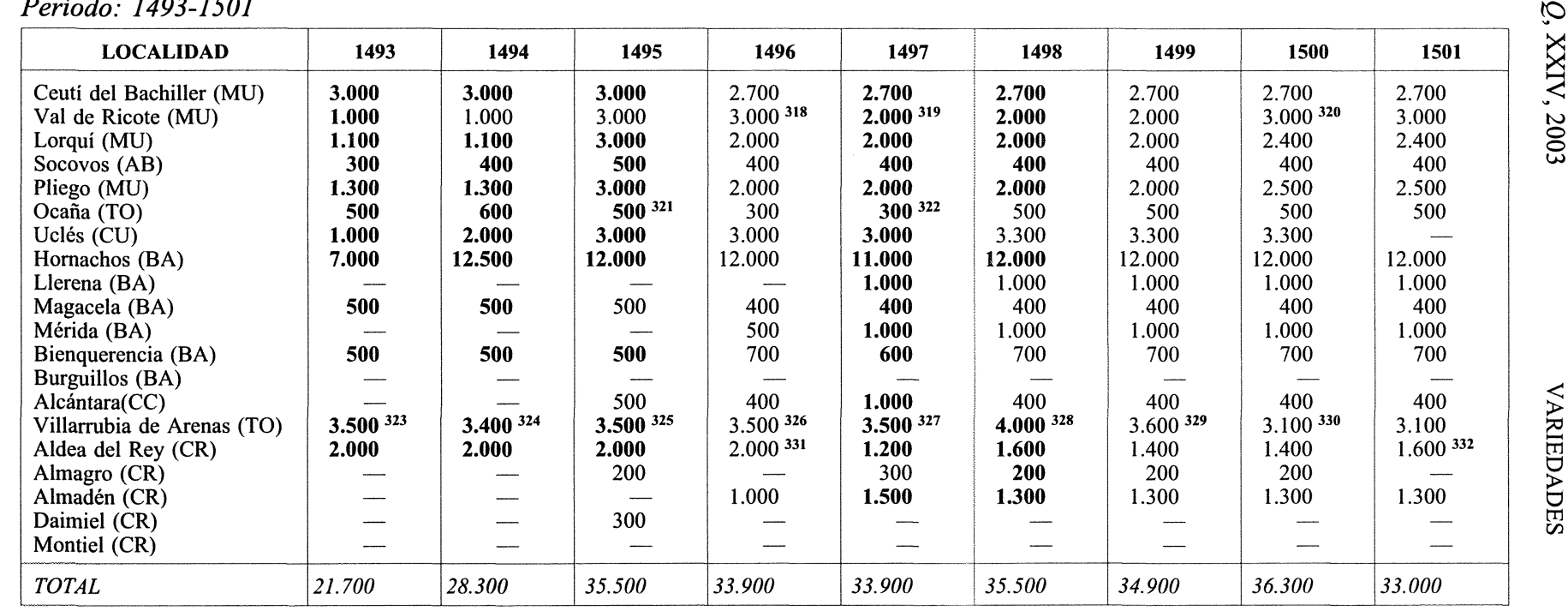

菖

頮 


\section{NOTAS A LAS TABLAS}

${ }^{1}$ Con Bonilla (provincia, en adelante, prov., de Ávila)

Idem.

${ }^{3}$ Con Bonilla (prov. de Ávila), e con los que con ellos suelen pechar.

${ }^{4}$ Con Béjar (prov. de Salamanca)

5 Idem.

${ }^{6}$ Con Béjar (prov. de Salamanca), e con los que con ellos suelen pechar.

7 Idem.

8 Idem.

9 Idem

${ }_{10}$ Idem.

${ }^{11}$ Idem.

${ }_{12}$ Idem.

${ }_{13}^{13}$ Idem.

${ }_{14}$ Con los que con ellos suelen pechar.

15 Idem.

16 Idem.

17 Idem.

18 Idem.

19 Idem.

20 Idem.

${ }^{21}$ Con el Real de Manzanares, Guadarrama (prov. de Madrid), y Sepúlveda (prov. de Segovia).

${ }_{22}$ Con el Real de Manzanares, Chinchón (prov. de Madrid), e con los que con ellos suelen pechar.

23 Idem.

${ }^{24}$ Idem.

${ }^{25}$ Idem.

${ }^{26}$ Con Sepúlveda (prov. de Segovia), el Real de Manzanares, y Chinchón (prov. de Madrid)

${ }^{27}$ Idem.

${ }^{28}$ Con Sepúlveda, Pedraza (prov. de Segovia), El Real de Manzanares, y Guadarrama (prov. de Madrid)

${ }^{29}$ Con Sepúlveda (prov. de Segovia), El Real de Manzanares, y Guadarrama (prov. de Madrid)
${ }^{30}$ Con Cuéllar (prov. de Segovia)
31 Idem.
32 Con los que con ellos suelen pechar.
${ }^{33}$ Idem.
${ }^{34}$ Idem.
${ }^{35}$ Con sus pecheros.
${ }^{36}$ Con Béjar (prov. de Salamanca)
${ }^{37}$ Con los que suelen pechar.
38 Idem.
39 Idem.
40 Idem.
41 Idem.
42 Idem.
43 Idem.
${ }^{44}$ Idem. 
45 Con Guadarrama, Manzanares (prov. de Madrid), Pedraza (prov. de Segovia), e con los que con ellos suelen pechar.

46 Idem.

${ }^{47}$ Con Guadarrama, Manzanares (prov. de Madrid), e con los que ellos suelen pechar.

${ }^{48}$ Con Guadarrama, Manzanares (prov. de Madrid), Pedraza (prov. de Segovia), e con los que con ellos suelen pechar.

${ }_{49}$ Con Guadarrama, Manzanares (prov. de Madrid), e con los que con ellos suelen pechar.

${ }^{50}$ Idem.

51 Con El Real de Manzanares, y Guadarrama (prov. de Madrid)

52 Con los moros de Peñafiel (prov. de Valladolid)

53 Con Manzanares (prov. de Madrid)

${ }_{54}$ Con Bustillo (prov. de Burgos)

55 Idem.

${ }^{56}$ Idem.

57 Idem.

58 Idem.

59 Idem.

60 Idem.

${ }^{61}$ Con los que con ellos suelen pechar.

62 Idem.

63 Idem.

${ }^{64}$ Con Coruña del Conde (prov. de Burgos)

${ }^{65}$ Idem.

66 Idem.

${ }^{67}$ Idem.

68 Idem.

${ }^{69}$ Idem.

${ }^{70}$ Idem.

${ }^{71}$ Con Arnedo y Préjano (prov. de La Rioja)

72 Idem.

${ }^{73}$ Con Arnedo y Préjano (prov. de La Rioja), e con los que con ellos suelen pechar.

74 Idem.

75 Idem.

76 Con Arnedo (prov. de La Rioja), e sus pecheros.

77 Idem.

78 Idem.

79 Con Préjano (prov. de La Rioja)

${ }^{80}$ Idem.

81 Con Bozoo (prov. de Burgos), Castañares y Bañares (prov. de La Rioja)

${ }^{82}$ Idem.

83 Idem.

${ }^{84}$ Idem.

${ }^{85}$ Idem.

86 Idem.

87 Idem.

88 Idem.

89 Con Belorado (prov. de Burgos)

90 Idem.

91 Idem.

92 Idem.

93 Idem. 
94 Idem.

${ }^{95}$ Idem.

96 Idem.

97 Idem.

98 Con Herce (prov. de La Rioja) Como se puede observar Herce ya contribuye por separado. Nos hemos limitado a transcribir el original tal cual, y en él se indica primero la aljama de moros de "Arze", y luego, nueve líneas más abajo, la aljama de moros de Préjano e "Yerçe". Pensamos que se debe a una confusión del escribano que ha apuntado una misma localidad dos veces.

${ }^{99}$ Idem.

100 Idem.

101 Idem.

102 Con Préjano (prov. de La Rioja)

103 Idem.

104 Idem.

105 Con los de Vaños (desconocemos el nombre actual de esta localidad)

106 Con Yepes, Los Yébenes, y La Puebla de Montalbán (prov. de Toledo)

107 Idem.

108 Con Yepes, Los Yébenes, y La Puebla de Montalbán (prov. de Toledo), e con los que con ellos suelen pechar.

109 Idem.

110 Idem.

111 Idem.

112 Con Yepes, y Los Yébenes (prov. de Toledo)

113 Con Yepes, Los Yébenes, y La Puebla de Montalbán (prov. de Toledo)

114 Idem.

115 Idem.

116 Con Valdemoro (prov. de Madrid)

117 Idem.

${ }_{118}$ Con Valdemoro (prov. de Madrid), e con los que con ellos suelen pechar.

119 Idem.

120 Idem.

121 Idem.

122 Idem.

123 Idem

124 Idem.

125 Con Barajas, Alcobendas, Pinto, y Móstoles (prov. de Madrid)

126 Idem. pechar.

${ }^{127}$ Con Barajas, Alcobendas, Pinto (prov. de Madrid), e con los que con ellos suelen

${ }^{128}$ Idem.

129 Idem.

${ }^{130}$ Idem.

131 Idem.

132 Idem.

133 Con Barajas (prov. de Madrid)

134 En el documento original transcribo, con Baxares y Tordegálvez. He considerado que el escribano quería referirse a los pueblos de Barajas y Torrelaguna (prov. de Madrid)

${ }_{135}$ Con Torrelaguna (prov. de Madrid), Talamanca, Uceda, y Mondéjar (prov. de Guadalajara)

136 Idem. 
137 Con Torrelaguna (prov. de Madrid), Talamanca, Uceda, Mondéjar (prov. de Guadalajara), e con los que con ellos suelen pechar.

138 Idem.

139 Idem.

140 Idem

141 Idem.

142 Idem.

143 Con Torrelaguna (prov. de Madrid)

${ }_{144}$ Con Brihuega, Pastrana, y Tendilla (prov. de Guadalajara)

145 Idem.

${ }^{146}$ Con Brihuega, Pastrana, Tendilla (prov. de Guadalajara), e con los que con ellos suelen pechar.

147 Idem.

148 Idem

149 Idem.

150 Idem.

151 Con Brihuega, y Pastrana (prov. de Guadalajara)

152 Con Oropesa (prov. de Toledo)

153 Idem.

154 Idem.

155 Idem.

156 Idem.

157 Idem.

158 Idem.

159 Idem.

160 Con Trijueque (prov. de Guadalajara)

161 Idem.

162 Con Trijueque (prov. de Guadalajara), e con los que con ellos suelen pechar.

163 Idem.

164 Idem

165 Idem.

166 Idem.

167 Idem.

168 Idem.

169 Con Valdolivas, Albendea (prov. de Cuenca), Salmerón, Alcocer, y Pareja (prov. de Guadalajara)
170 Idem.
171 Idem
172 Idem.
173 Idem.
174 Idem
175 Con Valdeolivas, Albendea (prov. de Cuenca), Salmerón, y Alcocer, (prov. de

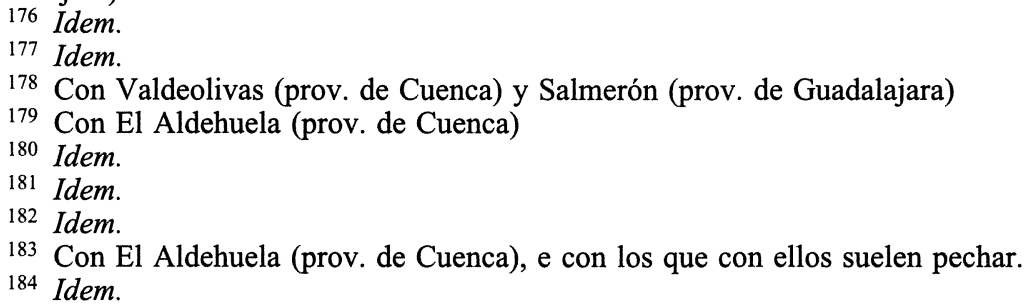
Guadalajara) 
185 Idem.

186 Idem.

187 Idem.

188 Con Zafra, Alconchel, Montalbán y Montalbanejo (prov. de Badajoz)

189 Idem.

190 Idem.

191 Con Zafra, Montalbán, y Montalbanejo (prov. de Badajoz)

192 Idem.

193 Idem.

194 Con Zafra, y Montalbanejo (prov. de Badajoz)

195 Con Zafra (prov. de Badajoz)

196 Con Zafra, y Montalbanejo (prov. de Badajoz)

197 Con ¿Musco? (desconocemos el nombre actual de dicha localidad)

198 Con Santa María del Campo, y Valverde (prov. de Cuenca)

199 Idem.

200 Idem.

201 Idem.

202 Idem.

203 Idem.

204 Idem.

205 Idem.

206 Idem.

207 Con Santa María del Campo (prov. de Cuenca)

208 Con Yepes, Los Yébenes, y La Puebla de Montalbán (prov. de Toledo)

209 Idem.

210 Idem.

211 Idem.

212 Idem.

213 Idem.

214 Idem.

215 Idem.

216 Idem.

217 Con Borox, el moro de Valdemoro (prov. de Madrid), e los que con ellos suelen pechar.

218 Con Borox, y el moro de Valdemoro (prov. de Madrid)

219 Con Borox, y el moro de Valdemoro (prov. de Madrid), e con los que con ellos suelen pechar.

220 Idem.

221 Con los que con ellos suelen pechar.

222 Idem.

223 Con Borox (prov. de Toledo), y Valdemoro (prov. de Madrid)

224 Con Barajas, y Torrelaguna (prov. de Madrid)

225 Idem.

226 Con Barajas, Pinto, Alcobendas (prov. de Madrid), y los que con ellos suelen pechar.

227 Idem.

228 Idem.

229 Idem.

230 Con los que con ellos suelen pechar.

231 Idem.

232 Con Barajas, Alcobendas, y Pinto (prov. de Madrid)

233 Con los que con ellos suelen pechar. 
234 Idem.

235 Idem.

236 Idem.

237 Con los que con ellos suelen pechar.

238 Los moros que solían pechar con Alcalá de Henares (prov. de Madrid), que es los dos moros de Cobeña (prov. de Madrid), e el moro de Algete (prov. de Madrid), y el moro de Fuentelsaz (prov. de Madrid), y el moro de Paracuellos (prov. de Madrid), y el moro de Talamanca (prov. de Madrid)

239 Idem.

240 Con los que con ellos suelen pechar.

241 Idem.

242 Idem.

243 Idem.

244 Idem.

245 Idem.

246 Idem.

247 Con Brihuega, Pastrana y Tendilla (prov. de Guadalajara)

248 Con Trijueque (prov. de Guadalajara)

249 Idem.

250 Idem.

251 Idem.

252 Idem.

253 Idem.

254 Idem.

255 Idem.

256 Idem.

257 Con Salmerón (prov. de Guadalajara), y Valdeolivas (prov. de Cuenca)

258 Idem.

259 Idem.

260 Idem.

261 Idem.

262 Idem.

263 Idem.

264 Idem.

265 Idem.

266 Con San Clemente (prov. de Cuenca)

267 Con los que con ellos suelen pechar.

268 Con Cáceres (prov. de Cáceres)

269 Con Las Garrovillas (prov. de Cáceres)

270 Idem.

271 Idem.

272 Idem.

273 Idem.

274 Idem.

275 Idem.

276 Idem.

277 Con los que con ellos suelen pechar.

278 Idem.

279 Idem.

280 Idem.

281 Con Cáceres (prov. de Cáceres), e con los que con ellos suelen pechar.

282 Idem. 
283 Idem.

${ }^{284}$ Idem.

285 Idem.

${ }^{286}$ Idem.

287 Idem.

288 Idem.

289 Con los que con ellos suelen pechar.

290 Idem.

291 Con Écija (prov. de Sevilla)

292 Con Mecergelo (lugar de Micer Egidio Bocanegra, en la provincia de Córdoba cercano a Palma del Río a la que los documentos denominan, Palma de Mecergelo)

293 Idem.

${ }^{294}$ Con Arrixaca (prov. de Murcia)

295 Idem.

${ }^{296}$ Idem.

297 Idem.

${ }^{298}$ Con Socovos (prov. de Albacete)

${ }^{299}$ Con Cotillas (prov. de Murcia)

${ }^{300}$ Idem.

301 Idem.

${ }^{302}$ Idem

303 Con El Palomar (de Juan Vicente) (prov. de Murcia)

304 Idem.

305 Idem.

306 No hay moros, salvo uno pobre.

307 No hay moros, salvo dos o tres, echámosles.

308 Con Blanca (prov. de Murcia)

309 Con Ribera (del Fresno) (prov. de Badajoz)

310 Con Alcázar de Consuegra, y Daimiel (prov. de Ciudad Real)

311 Idem.

312 Idem.

313 Idem.

314 Idem.

315 Idem.

316 Idem.

${ }^{317}$ Idem.

318 Hace referencia a los moros de seis lugares del Val de Ricote.

319 Idem.

${ }^{320}$ Idem.

321 Con Dos Barrios (prov. de Toledo)

${ }^{322}$ Idem.

${ }^{323}$ Con los que con ellos pechan.

${ }^{324}$ Idem.

325 Idem.

${ }^{326}$ Idem.

327 Con los moros de Daimiel (prov. de Ciudad Real), e con los que con ellos suelen pechar.

${ }^{328}$ Con los que suele pechar

329 Idem.

330 Idem.

331 Con Almagro (prov. de Ciudad Real)

332 Idem. 